\title{
Efficacy and Safety of Loxoprofen Hydrogel Transdermal Patch Versus Loxoprofen Tablet in Chinese Patients with Myalgia: A Double-Blind, Double-Dummy, Parallel-Group, Randomized, Controlled, Non-Inferiority Trial
}

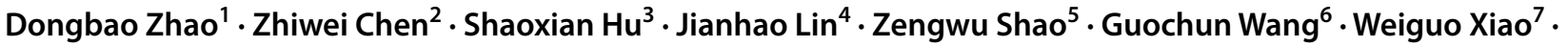 \\ Yi Zheng ${ }^{8} \cdot$ Zhiyi Zhang $^{9} \cdot$ Yeqing Shi ${ }^{1} \cdot$ Zhanguo $\mathrm{Li}^{10}$
}

Published online: 6 February 2019

(c) The Author(s) 2019

\begin{abstract}
Background and Objective Loxoprofen (LOX) is a nonsteroidal anti-inflammatory drug (NSAID). Although oral administration of LOX has been widely prescribed, clinical guidelines for osteoarthritis generally recommend topical rather than oral NSAIDs in specific patients. However, there is limited information on the effects of loxoprofen sodium oral (LOX-O) versus loxoprofen sodium hydrogel transdermal patch (LOX-T) in myalgia patients. Hence, this non-inferiority study was designed to compare the efficacy and safety of LOX-O versus LOX-T in Chinese patients with myalgia.

Methods In this double-blind, double-dummy, parallel-group, randomized controlled trial, 182 Chinese patients were enrolled and randomized equally to either LOX-T or LOX-O treatment for 2 weeks. Patients in the LOX-T group applied one sheet of the active LOX-T once a day on the affected site and took one placebo tablet three times a day immediately after meals, whereas patients in the LOX-O group applied one sheet of the placebo patch once a day and took one active LOX-O three times a day. Primary endpoint was the proportion of patients with 50\% overall improvement or higher at the final visit. The cutoff value of a non-inferiority difference was set as $-10 \%$.

Results In the full analysis set, the primary endpoint of final efficacy rate was $81.3 \%(n=91)$ in the LOX-T group and $72.2 \%$ $(n=88)$ in the LOX-O group. The difference between the two groups was 9.1\% [95\% confidence interval (CI) -3.1 to $21.3 \%$ ], which showed that LOX-T was non-inferior compared with LOX-O. No serious adverse events occurred in either group. Conclusions This trial showed the non-inferiority of LOX-T compared with LOX-O in efficacy and safety in Chinese patients with myalgia. Also, the characteristic features of topical LOX-T, such as better compliance and lower risk-benefit ratio, make it more favorable for clinical practice.
\end{abstract}

Trial Registration The study was registered in the isrctn.com registry (ISRCTN trial ID: ISRCTN16227145).

\section{Key Points}

We compared the efficacy and safety of loxoprofen sodium oral (LOX-O) with sodium hydrogel transdermal patch (LOX-T) in Chinese patients with myalgia.

The trial showed the non-inferiority of LOX-T compared with LOX-O in efficacy and safety in Chinese patients with myalgia.

Zhanguo Li

lzg20140310@sina.com

Extended author information available on the last page of the article

\section{Introduction}

Myalgia is a symptom, usually defined as pain often associated with injury, including trauma, surgery, musculoskeletal injuries such as strains, sprains, and overuse injuries, or soft tissue injuries such as muscle soreness or cramps [1-5]. Nonsteroidal anti-inflammatory drugs (NSAIDs) are widely used in the treatment of pain and inflammation associated with chronic conditions caused by myalgia [6]. NSAIDs are classified into selective (COX-2 inhibitors) and nonselective inhibitors based on their mode of action. Although oral NSAIDs are safe and effective, topical NSAIDs offer similar efficacy to the oral route for relief with improved safety profile due to their reduced systemic absorption [6]. 
Clinical guidelines for osteoarthritis generally recommend topical rather than oral NSAIDs, particularly in patients aged $>75$ years [3, 7], and in those with comorbidities or an increased risk of gastrointestinal, cardiovascular, or renal adverse effects [7].

Loxoprofen (LOX) is a prodrug of phenyl mefenamic acid, a nonselective NSAID, developed with an assumption that it would be associated with fewer NSAID-related adverse events (AEs) [1, 8]. Oral administration of LOX has been widely prescribed for pain in Japan and China [1]. Topical NSAIDs such as the LOX sodium hydrogel transdermal patch (LOX-T) was developed to control pain while lowering the risk of side effects [4, 9-11]. LOX penetrates directly into the affected site through the topical route, resulting in consistent and safe pain relief. Animal studies have shown that skin-absorbed LOX can be metabolized to the active trans-OH compound, similar to oral formulations [9]. Although clinical guidelines recommend the use of NSAIDs or topical NSAIDs for pain relief, there are limited data available to assess the effects of LOX-T versus loxoprofen sodium oral (LOX-O). Thus, this double-blind, double-dummy, multicenter, non-inferiority randomized controlled trial was designed to evaluate the efficacy and safety of LOX-T compared with LOX-O in Chinese patients with myalgia from all causes.

\section{Patients and Methods}

\subsection{Study Participants}

Patients suffering from myalgia from all causes, aged between 18 and 80 years, and presenting with tenderness, rest pain, or motion pain were enrolled. Prior to randomization, a washout period of at least 1 week was mandatory in patients taking painkillers. It was also required that there was no change in physical therapy within 2 weeks prior to initiation of enrollment for the trial.

Exclusion criteria included the following: (1) acute traumatic pain; (2) neurological symptoms; (3) poor local skin condition; (4) required combination therapy with steroids or other NSAIDs; (5) chronic diseases, such as peptic ulcer, asthma, unstable cardiac disease, renal or hepatic dysfunction, hematologic disorders, diabetes, psychosis, and other complications; (6) pregnancy, lactation, or preparing for fertilization; (7) hypersensitivity history to LOX or similar medications; (8) participation in other clinical trials within 3 months.

\subsection{Trial Design}

Fifteen rheumatological or orthopedic centers (covering north, east, central south and north-east regions) in China contributed independently to this double-blind, doubledummy, non-inferiority, and head-to-head randomized controlled trial (RCT) from June 2010 to October 2011. After screening, patients were randomly assigned $(1: 1)$ to either the LOX-T group [LOX-T (Lead Chemical Co., Ltd.) plus tablet placebo] or the LOX-O group [LOX-O (Daiichi Sankyo Co., Ltd.) plus patch placebo]. The patch placebo contained the same ingredients as LOX-T apart from LOX, and had physical characteristics similar to LOX-T. The tablet placebo also had the same ingredients as LOX-O apart from LOX with physical characteristics similar to those of LOXO. During the 2-week trial, participants in the LOX-T group were instructed to apply LOX-T onto clean skin overlying the affected muscles every night and fix it with a reticular bandage for 24 hours. They simultaneously took a placebo tablet three times a day after meals. Those assigned to receive the patch placebo applied it in a similar manner and received the active LOX-O three times a day after meals. LOX-T contained $100 \mathrm{mg}$ active LOX per unit, proven by previous studies to be optimal, while LOX-O contained $60 \mathrm{mg}$ active LOX per unit, thus making total oral exposure reach $180 \mathrm{mg}$ daily $[1,11]$.

The demographic characteristics and clinical, laboratory, and radiographic assessments of the subjects were recorded at baseline. Clinical and laboratory variables, drug compliance, AEs, overall improvement, and efficacy rates were evaluated at Week 1 and 2.

The study was registered in the isrctn.com registry (ISRCTN trial ID: ISRCTN16227145). The study protocol and research manual were approved by the Ethics Committees of all institutions involved. Written informed consent was obtained from all subjects in accordance with the Declaration of Helsinki principles and the guidelines for Good Clinical Practice of the International Conference on Harmonization.

\subsection{Sample Size}

The sample size was calculated based on Phase III study results in Japan [12]. Considering the efficacy rate of LOX-T (75.2\%) and LOX-O (64.5\%) at week 2, a non-inferiority margin $-10 \%$ was set [13]. Considering a type I error rate of $2.5 \%$ (one-tailed) and $80 \%$ power, a sample size of 77 patients per group was required to show the non-inferiority. It was increased to at least 80 patients per group to account for attrition.

\subsection{Randomization and Blinding}

Randomized assignments were stratified according to the trial center. A computer-generated block randomization list was maintained by individuals who were strictly forbidden to discuss the particulars of treatment with investigators and 
patients involved in the trial. Each block consisted of only four patients to avoid imbalances in treatment allocation at each clinical center. Patients were sequentially assigned their randomization number at each center, and the individual code was kept in single-sealed opaque envelopes to be opened only in case of a medical emergency. The participants, caregivers, and those assessing the outcomes were all blinded to group assignment.

\subsection{Efficacy and Safety Assessments}

The primary endpoint was defined as overall efficacy rate at Week 2. Based on four major myalgia symptoms and by using "last observation carried forward" method in the full analysis set, data from the last visit in early termination patients were also included. The symptoms included severity of pain at rest, pain on motion, and tenderness, all of which were classified using a 4-grade scale: 0: no symptoms, 1: mild, 2: moderate, and 3: severe. The fourth symptom, disability in daily activities, was defined as 0: not confined, $1: 75 \%$ to $99 \%$ of normal, $2: 50 \%$ to $74 \%$ of nor$\mathrm{mal}$, and $3:<50 \%$ of normal. In addition, the symptomatic scores were summated at each visit to provide a total score of clinical symptoms. The overall improvement percentage was calculated by (1: total score of clinical symptoms at visit/ total score of clinical symptoms at baseline) $\times 100 \%$. Finally, the overall efficacy rate was calculated as the proportion of patients with 50\% overall improvement or higher.

The secondary endpoint was symptomatic efficacy rates. Efficacy rates of the four symptoms were evaluated at Week 2 separately, defined as the proportion of patients at Week 2, who scored lower than at baseline. The safety assessment in randomized patients who received at least one dose of study drugs and were included as the safety set. All events were recorded under the guidelines of the Medical Dictionary for Regulatory Activities. Routine laboratory tests, including whole blood count, complete chemistry panel, and urinalysis, were carried out as per protocol at baseline and Week 2 .

\subsection{Statistical Analysis}

Non-inferiority of final overall efficacy rate of LOX-T versus LOX-O was evaluated if the lower limit of the $95 \% \mathrm{CI}$ for the difference in efficacy rate was not less than the cutoff value of $-10 \%$ based on previous studies $[12,14]$. The comparison between the groups was performed for overall efficacy rate at Week 1 and Week 2 and the total score of clinical symptoms at first, second, and final visits using unpaired Student's $t$ test (one-sided) for non-inferiority comparison or the Wilcoxon-Mann-Whitney, test depending on the normality and heterogeneity. A $p$ value of $<0.05$ was considered statistically significant. The overall improvement rate at endpoint was analyzed using an analysis of covariance
(ANCOVA) model with a factor for treatment and baseline total score of clinical symptoms, center, treatment $\mathrm{x}$ center as covariates. The point estimate (the least squares means) and two-sided 95\% CI based on the ANCOVA model was provided for the difference between the treatment groups. Incidence of AEs, serious adverse events, and non-expected events were compared between two groups using the Pearson Chi-square test and Fisher's exact model. A per-protocol (PP) complete analysis was also performed which included only those patients who completed the treatment that was originally allocated to them. All statistics were analyzed by SPSS 18.0.

\section{Results}

\subsection{Study Population and Baseline Characteristics}

A total of 182 eligible patients were enrolled and randomized between June 2010 and October 2011. Two patients in the LOX-T group and five patients in the LOX-O group ( $2.2 \%$ and $5.5 \%$, respectively) did not complete the 2 -week treatment as required by the study protocol, resulting in an overall dropout rate of $3.8 \%$ (Fig. 1).

Due to lack of data after randomization, one patient in the LOX-O group was excluded immediately and thus the full analysis set comprised 91 patients in the LOX-T group and 88 patients in the LOX-O group. All randomized patients who received at least one dose of study drugs were included in the safety set (Fig. 1).

The demographics and clinical characteristics at baseline in the full analysis set did not show significant difference between the two study groups except for pain at rest and total score of clinical symptoms (Table 1).

\subsection{Compliance of Study Drugs}

The average compliance for topical administration, patches of either LOX or placebo, was approximately $98 \%$, while the average compliance for tablets was only about $88 \%$ to $92 \%$. No statistically significant difference was reported between the two groups (data not shown).

\subsection{Primary Efficacy Endpoint}

The results of overall efficacy rates at Week 1,2, and final visit in modified full analysis set population was evaluated. The primary endpoint of efficacy rate at final visit was $81.3 \%(n=91)$ in the LOX-T group and $72.7 \%(n=88)$ in the LOX-O group. The difference between the two groups was $8.6 \%$ (95\% CI -3.7 to $-20.9 \%$ ), comparing non-inferiority of LOX-T with that of LOX-O. The overall efficacy rates in PP analysis set in both groups were similar $(82.1 \%, n=69$, 
Fig. 1 Study subject contexture. $F A S$ full analysis set, $L O X-O$ loxoprofen sodium oral, $L O X-T$ loxoprofen sodium transdermal patch

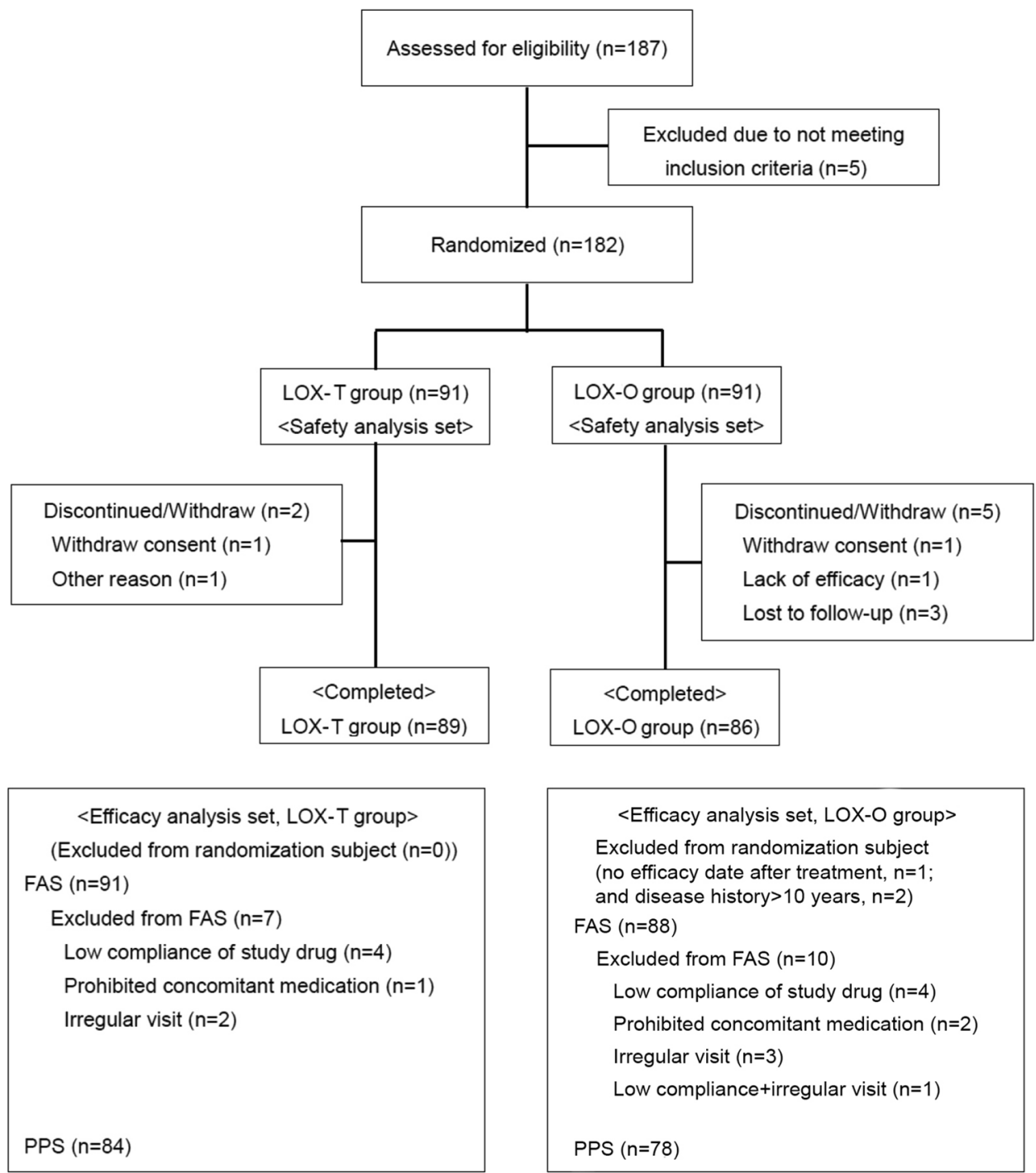

95\% CI 74.0-90.3\% vs 76.9\%; $n=60,95 \%$ CI 67.6-86.3\%; $\mathrm{p}=0.410$, a difference of $5.2 \%, 95 \% \mathrm{CI}-7.2$ to $17.6 \%$ ). Based on the result of covariance analysis, baseline total score of clinical symptoms was not significant $(p=0.400)$ (Table 2). Besides, adjusted treatment difference was 4.723 (95\% CI 4.2-13.6), while the raw treatment difference and 95\% CI was 2.8 ( -4.6 to $10.2 \%$ ) (Table 3 ). The baseline total score of clinical symptoms was not a significant factor for the overall improvement rate.

\subsection{Secondary Efficacy Endpoints}

Changes in secondary efficacy endpoints (rest pain, tenderness, pain on motion, and disability in daily activities) were compared between the LOX-T and LOX-O groups at final visit. No significant differences were found in the full analysis set analyses between the two groups ( $p>0.05)$ (Fig. 2). The differences in all outcomes were above the predetermined non-inferiority margin $(-10 \%)$, except for pain on motion, and with a $95 \% \mathrm{CI}$ of -12.2 to $10.9 \%$ in the full analysis set.

\subsection{Safety}

The total incidence of AEs reported during treatment were $14.3 \%(n=13 / 91)$ in the LOX-T group and $22.0 \%(n=20 / 91)$ in the LOX-O group, with $p=0.178$. There were no serious adverse events in either the LOX-T group or the LOX-O group. The most frequent AEs were gastrointestinal complaints (e.g. abdominal discomfort, abdominal pain, diarrhea, and oral pain) (5.5\%, five patients in the LOX-T group; 7.7\% and seven patients in the LOX-O group). Infections were also common, with 3.3\% reported in both the LOX-T and LOX-O groups. Other AEs were sporadic, including skin, muscleskeletal, cardiac, pulmonary, metabolic, menstrual, or psychological problems, as summarized in Table 4. 
Table 1 Demographics and baseline clinical characteristics of patients in modified FAS population ${ }^{\mathrm{a}}$

\begin{tabular}{|c|c|c|c|}
\hline Characteristics & $\begin{array}{l}\text { LOX-T } \\
n=91\end{array}$ & $\begin{array}{l}\text { LOX-O } \\
n=88\end{array}$ & $p$ value \\
\hline Age, years (\%) & $45.6(13.9)$ & $46.4(14.1)$ & 0.700 \\
\hline Female, $n(\%)$ & $56(61.5)$ & $52(59.1)$ & 0.738 \\
\hline Height, cm (\%) & $164.5(8.5)$ & $165.2(8.0)$ & 0.423 \\
\hline Weight, $\mathrm{kg}(\%)$ & $63.4(11.8)$ & $64.7(11.4)$ & 0.476 \\
\hline BMI, $\mathrm{kg} / \mathrm{cm}^{2}(\%)$ & $23.3(3.2)$ & $23.6(3.0)$ & 0.552 \\
\hline Affected sites, $n(\%)$ & & & 0.808 \\
\hline Neck & $14(15.4)$ & $10(11.4)$ & \\
\hline Shoulders & $17(18.7)$ & $14(15.9)$ & \\
\hline Arms & $4(4.4)$ & $2(2.3)$ & \\
\hline Back and waist & $38(41.8)$ & $45(51.1)$ & \\
\hline Legs & $14(15.4)$ & $15(17.0)$ & \\
\hline Buttocks & $1(1.1)$ & $0(0.0)$ & \\
\hline Other & $3(3.3)$ & $2(2.3)$ & \\
\hline Duration of myalgia (d) & & & 0.905 \\
\hline Mean (SD) & $4.6(11.6)$ & $8.5(22.2)$ & \\
\hline Median (min-max) & $1.0(1-78)$ & $1.0(1-98)$ & \\
\hline Allergy history, $n(\%)$ & 0 & 0 & \\
\hline Comorbidity, $n(\%)$ & $11(12.1)$ & $13(14.8)$ & 0.598 \\
\hline Medical/surgical history, $n(\%)$ & $15(16.5)$ & $17(19.3)$ & 0.621 \\
\hline \multicolumn{4}{|l|}{ Clinical symptoms, $n(\%)$} \\
\hline Pain at rest & & & 0.049 \\
\hline No symptom & $12(13.2)$ & $16(18.2)$ & \\
\hline Mild & 37 (40.7) & $45(51.1)$ & \\
\hline Moderate & $40(44.0)$ & $25(28.4)$ & \\
\hline Severe & $2(2.2)$ & $2(2.3)$ & \\
\hline \multicolumn{4}{|l|}{$\begin{array}{l}\text { Clinical symptom score at } \\
\text { baseline, mean (SD) }\end{array}$} \\
\hline Rest pain & $1.4(0.7)$ & $1.1(0.7)$ & 0.049 \\
\hline Tenderness & $1.7(0.7)$ & $1.5(0.8)$ & 0.251 \\
\hline Motion pain & $1.9(0.5)$ & $1.8(0.6)$ & 0.798 \\
\hline Total score & $6.4(1.6)$ & $5.8(1.7)$ & 0.010 \\
\hline
\end{tabular}

$B M I$ body mass index, FAS full analysis set, $S D$ standard deviation

${ }^{a}$ Modified FAS population excluded subjects who had history of myalgia for $>10$ years; two subjects were excluded from LOX-O study group

Routine laboratory examinations indicated that no patients in the LOX-T group developed abnormalities. Four patients $(4.4 \%)$ in the LOX-O group showed mild or moderate changes in hematological examinations.

\section{Discussion}

The current myalgia guidelines support the use of NSAIDs as first-line therapeutic agents for symptomatic relief of various inflammatory conditions and myalgia [15-17]. Despite growing evidence, definite replacement of oral NSAIDs by topical route as an alternate option is yet to see clinical success. In these terms, the current double-dummy, doubleblind RCT has shown that LOX-T is not inferior to LOX-O with regard to efficacy, and its safety profile is also favorable with no serious adverse events being observed. These observations strongly support the existing evidence that LOX-T is a potential alternative to LOX-O.

The current findings showed that LOX-T was effective in $81.3 \%$ of patients compared with $72.7 \%$ of those in the LOX-O group. These results are in line with findings from double-blind trials, which assessed the overall improvement in clinical symptoms of once-daily LOX $100 \mathrm{mg}$ hydrogel patches versus oral LOX $60 \mathrm{mg}$ three times daily in patients with knee osteoarthritis, myalgia, or trauma-induced swelling and pain $[12,14,18-21]$. Most patients in both treatment groups (60-98\%) had $>50 \%$ of overall improvement in clinical symptoms. Furthermore, LOX-T patches were noninferior to ketoprofen patches as seen in patients with knee osteoarthritis over 4 weeks and indomethacin patches as seen in patients with myalgia with regard to the final overall improvement rate [22, 23]. In Japan, post-marketing studies have shown that LOX-T patches and tape are associated with high rates of overall clinical improvement (>95\%) among patients with osteoarthritis, myalgia, or trauma-induced swelling and pain $[24,25]$.

Topical NSAIDs are well known for their role in effectively decreasing both acute and chronic pain by inhibiting prostaglandin synthesis and thereby decreasing the inflammatory responses. Similar efficacy of transdermal patch to that of oral LOX seen in the current study and other studies is due to the similar mechanism of action, that is, inhibition of prostaglandin synthesis. These mechanisms are further strengthened by the fact that as a prodrug, LOX is metabolized by carbonyl-reductase in local tissues including skin and liver to form trans-OH form of active metabolite [26]. Therefore, drug concentration of active metabolite is high only in local, affected areas for 24 hours and low in blood [27]. Although a similar study has been conducted by $\mathrm{Mu}$ et al. [14], the key difference between the current and former study lies in assessing the efficacy of LOX-T versus LOX-O in Chinese patients with knee osteoarthritis, whereas our study included patients suffering from myalgia from all causes. This criterion also enabled enrollment of patients from a wide age range (18-80 years) unlike the aforementioned trial which consisted of patients aged $>40$ years owing to the disease condition.

The variances between the LOX-T and LOX-O groups in pain at rest and total score of clinical symptoms could result from the inevitable differences among centers. A covariance analysis was performed to adjust the overall improvement rate. However, the difference between the LOX-T and LOX-O groups was still higher than $-10 \%$, as presented in Tables 2 and 3, validating the non-inferiority between 
Table 2 Covariance analysis of overall improvement rate of two groups at endpoint (FAS)

\begin{tabular}{lccccc}
\hline Source & Degree of freedom & $\begin{array}{l}\text { Sum of square (SS) } \\
\text { value }\end{array}$ & $\begin{array}{l}\text { Mean square (MS) } \\
\text { value }\end{array}$ & $F$ value & $p$ value \\
\hline Model & 18 & 37972.548 & 2109.586 & 4.48 & $<0.001$ \\
Error & 162 & 76289.972 & 470.926 & & 0.400 \\
Corrected total & 180 & 114262.520 & & & 0.299 \\
Baseline values & 1 & 334.775 & 334.775 & 0.711 & 1.088 \\
$\begin{array}{l}\text { Randomized study } \\
\quad\end{array}$ & 1 & 512.292 & 512.292 & & $<0.001$ \\
$\quad \begin{array}{l}\text { groups } \\
\text { Study sites }\end{array}$ & 8 & 35312.712 & 4414.089 & 9.373 & 0.906
\end{tabular}

Table 3 Corrected means and 95\% confidence intervals (CIs)

\begin{tabular}{lrcl}
\hline Group & Mean & $\begin{array}{l}\text { Upper limit of } \\
95 \% \text { CI }\end{array}$ & $\begin{array}{l}\text { Lower limit } \\
\text { of 95\% CI }\end{array}$ \\
\hline LOX-T & 63.097 & 57.214 & 68.979 \\
LOX-O & 58.374 & 51.775 & 64.973 \\
$\begin{array}{l}\text { Difference between two } \\
\text { groups }\end{array}$ & 4.723 & -4.219 & 13.666 \\
\hline
\end{tabular}

LOX-T and LOX-O. Besides, in the current study, patients developed other complications and an equal number of patients in both treatment groups commonly needed treatment with other medications such as calcium antagonists $(2.5 \%)$ and antidiabetic drugs (1.5\%). This could have probably introduced a bias to our analysis. However, the outcomes were constant even after excluding two patients with long-term disease.

Since LOX is a prodrug of a short-acting NSAID, it is known to be associated with less frequent AEs than the other NSAIDs. However, long-term administration of NSAIDs predisposes patients to increased risk of developing upper gastrointestinal complications such as gastrointestinal ulcers and bleeding, the most common AEs [28-30]. In our study, a decreasing trend of gastrointestinal events was seen in the LOX-T group compared with the LOX-O group. Topical reactions and skin or subcutaneous problems leading to mild skin irritation or photosensitivity were also observed. Four patients in the LOX-O group showed mild-to-moderate changes in hematological parameters, whereas LOX-T had none. These findings corroborate with results of another study [14], thereby suggesting that LOX-T is non-inferior to LOX-O in terms of safety profile, owing to a lower daily exposure. For patients needing long-term administration of NSAIDs, LOX-T $(100 \mathrm{mg}$ ) offers a safer AE profile because of its lower daily exposure when compared to LOX-O $(180 \mathrm{mg} ; 60 \times 3 \mathrm{mg} /$ day $)$. This could be explained by the fact that the topical formulation enables distribution of NSAID to the involved muscles locally and not systemically. Even in animal studies, subcutaneous LOX concentration in the LOX-T group was higher than that seen in the LOX-O group, suggesting that drug concentration of active metabolite is higher in treated tissues, and lower in blood after treatment with LOX-T, which might decrease the risk of systemic side effects [9]. Additionally, the average compliance as monitored in this study for transdermal patches was $98 \%$ and $88 \%$ to $92 \%$ for oral tablets, indicating the convenience in using the patch. Overall, the aforementioned results give LOX-T an edge over LOX-O in treating myalgia with better efficacy, safety and the convenience involved it its usage.

However, there were a few limitations in this study. Approximately $12.6 \%$ of the patients received a combination of other medications along with LOX for comorbidities during the study. This bias was however corrected by a well-balanced distribution of such patients between the two groups. Also, our study was not sufficiently powered to detect significant difference for low incidence of AEs between the two groups.
Fig. 2 Sub-analysis for symptom improvement rate at final visit in full analysis set population. $L O X-O$ loxoprofen sodium oral, $L O X-T$ loxoprofen sodium transdermal patch. $p$ values were based on Pearson Chi-square test between two study groups

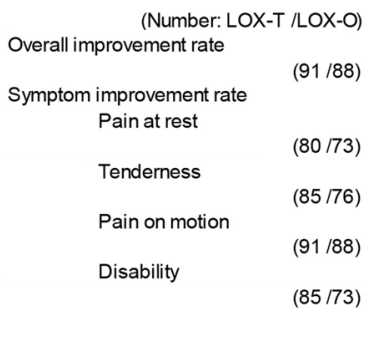

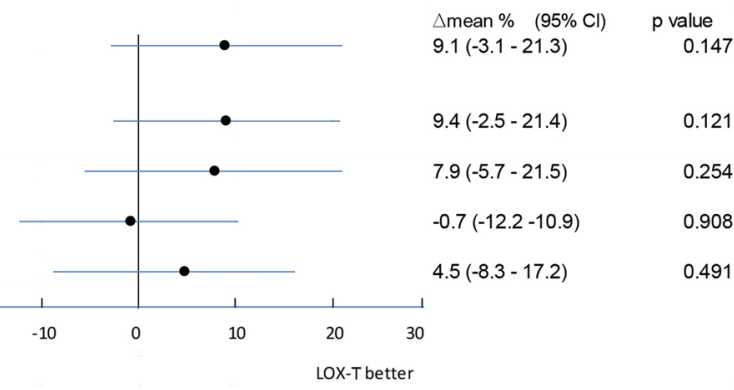

$-30-20$

LOX-Obetter 
Table 4 Summary of adverse events occurring in each group during treatment

\begin{tabular}{|c|c|c|c|}
\hline $\mathrm{AE}$ & $\begin{array}{l}\text { LOX-T group }(n=91) \\
n(\%)\end{array}$ & $\begin{array}{l}\text { LOX-O group }(n=91) \\
n(\%)\end{array}$ & $p$ value \\
\hline Any AEs & $13(14.3 \%)$ & $20(22.0 \%)$ & 0.178 \\
\hline Drug-related AEs ${ }^{\mathrm{a}}$ & $7(7.7 \%)$ & $14(15.4 \%)$ & 0.178 \\
\hline Gastrointestinal disorders & $5(5.5 \%)$ & $7(7.7 \%)$ & \\
\hline Abdominal discomfort & $4(4.4 \%)$ & $3(3.3 \%)$ & \\
\hline Abdominal pain & $1(1.1 \%)$ & $1(1.1 \%)$ & \\
\hline Diarrhea & $0(0.0 \%)$ & $3(3.3 \%)$ & \\
\hline Oral pain & $0(0.0 \%)$ & $1(1.1 \%)$ & \\
\hline Infections & $3(3.3 \%)$ & $3(3.3 \%)$ & \\
\hline Rhinopharyngitis & $2(2.2 \%)$ & $1(1.1 \%)$ & \\
\hline Upper respiratory tract infection & $1(1.1 \%)$ & $2(2.2 \%)$ & \\
\hline General disorders and administration site conditions & $2(2.2 \%)$ & $0(0.0 \%)$ & \\
\hline Discomfort & $1(1.1 \%)$ & $0(0.0 \%)$ & \\
\hline Inflammation & $1(1.1 \%)$ & $0(0.0 \%)$ & \\
\hline Skin or subcutaneous disorders & $2(2.2 \%)$ & $3(3.3 \%)$ & \\
\hline Erythema & $2(2.2 \%)$ & $1(1.1 \%)$ & \\
\hline Pruritus & $1(1.1 \%)$ & $1(1.1 \%)$ & \\
\hline Hidrosis & $0(0.0 \%)$ & $1(1.1 \%)$ & \\
\hline Cardiac system disorders & $1(1.1 \%)$ & $1(1.1 \%)$ & \\
\hline Tachycardia & $1(1.1 \%)$ & $0(0.0 \%)$ & \\
\hline Palpitation & $0(0.0 \%)$ & $1(1.1 \%)$ & \\
\hline Musculoskeletal/connective tissue diseases & $1(1.1 \%)$ & $1(1.1 \%)$ & \\
\hline Muscle fatigue & $1(1.1 \%)$ & $0(0.0 \%)$ & \\
\hline Ostealgia & $0(0.0 \%)$ & $1(1.1 \%)$ & \\
\hline Mental disorders & $1(1.1 \%)$ & $0(0.0 \%)$ & \\
\hline Insomnia & $1(1.1 \%)$ & $0(0.0 \%)$ & \\
\hline Respiratory system disorders & $1(1.1 \%)$ & $1(1.1 \%)$ & \\
\hline Cough & $1(1.1 \%)$ & $1(1.1 \%)$ & \\
\hline Laboratory tests & $0(0.0 \%)$ & $4(4.4 \%)$ & \\
\hline Decreased $\mathrm{Hb}$ & $0(0.0 \%)$ & $1(1.1 \%)$ & \\
\hline Hepatic dysfunction & $0(0.0 \%)$ & $1(1.1 \%)$ & \\
\hline Decreased platelet count & $0(0.0 \%)$ & $1(1.1 \%)$ & \\
\hline Decreased WBC count & $0(0.0 \%)$ & $2(2.2 \%)$ & \\
\hline Elevated WBC count & $0(0.0 \%)$ & $1(1.1 \%)$ & \\
\hline Metabolic or nutrition-related disorder & $0(0.0 \%)$ & $1(1.1 \%)$ & \\
\hline Reduced appetite & $0(0.0 \%)$ & $1(1.1 \%)$ & \\
\hline Nervous system disorders & $0(0.0 \%)$ & $1(1.1 \%)$ & \\
\hline Dizziness & $0(0.0 \%)$ & $1(1.1 \%)$ & \\
\hline Genital system disorder & $0(0.0 \%)$ & $1(1.1 \%)$ & \\
\hline Menstrual disorder & $0(0.0 \%)$ & $1(1.1 \%)$ & \\
\hline
\end{tabular}

AEs were reported according to the Medical Dictionary for Regulatory Activities (MedDRA) Version 14.1

${ }^{a}$ Drug-related AEs were determined by the investigator to be definitely, probably, or possibly medication related, and unknown relationship with medication. Patients were counted once for each unique AE and may have had more than one unique AE. $p$ value was evaluated using the Fisher's exact model. a: local AEs under and around patch. b: diffuse symptom at whole body 


\section{Conclusions}

In conclusion, this trial confirms the non-inferiority of topical LOX patches compared with oral LOX in terms of efficacy and safety for patients suffering from myalgia of any cause. Lower systemic exposure of the active drug, better compliance, and lower risk-benefit ratio makes topical LOX patches more favorable in practice. Thus, it provides supporting evidence towards approval of LOX-T as a firstline medication either alone or in combination with nonpharmacologic therapy to maximize the analgesic effect of the treatment.

Acknowledgements The study was funded by Daiichi Sankyo Co., Ltd. All authors expressed sincere gratitude to the patients and their families for their cooperation. Medical writing support under authors' direction was provided by Priyanka Bannikoppa, PhD and Amit Bhat, $\mathrm{PhD}$, CMPP (Indegene, Bangalore).

Author Contributions DZ, ZC, and SH were involved in data collection, statistical analysis, and drafting of the manuscript. JL, ZS, and GW designed the study, participated in data collection and statistical analysis, and revised the manuscript. WX, YZ, and ZZ participated in data collection. DZ, YS, and ZL participated in data collection and statistical analysis. All authors read and approved the final manuscript.

\section{Compliance with Ethical Standards}

Ethics approval and consent to participate The study protocol and research manual were approved by the Ethics Committees of all institutions involved. Written informed consent was obtained from all subjects in accordance with the Declaration of Helsinki principles and the guidelines for Good Clinical Practice of the International Conference on Harmonization.

Availability of data and materials The data sets used and/or analyzed during the current study are available from the corresponding author on reasonable request.

Conflict of interest The authors declare that they have no conflict of interest.

Funding The study was funded by Daiichi Sankyo Co., Ltd.

OpenAccess This article is distributed under the terms of the Creative Commons Attribution-NonCommercial 4.0 International License (http://creativecommons.org/licenses/by-nc/4.0/), which permits any noncommercial use, distribution, and reproduction in any medium, provided you give appropriate credit to the original author(s) and the source, provide a link to the Creative Commons license, and indicate if changes were made.

\section{References}

1. Sachiko S, Akio T, Mamoru T, Yoshikatsu K, Yukio I, Yoshio I, et al. Evaluation of administration frequency of LX-A in patients with osteoarthritis of the knee-randomized, open-label study compared between once-daily administration and twice-daily administration. J Clin Ther Med. 2006;22:311-26.

2. Burmester G, Lanas A, Biasucci L, Hermann M, Lohmander S, Olivieri I, et al. The appropriate use of non-steroidal anti-inflammatory drugs in rheumatic disease: opinions of a multidisciplinary European expert panel. Ann Rheum Dis. 2011;70:818-22.

3. Hochberg MC, Altman RD, April KT, Benkhalti M, Guyatt G, McGowan J, et al. American College of Rheumatology 2012 recommendations for the use of nonpharmacologic and pharmacologic therapies in osteoarthritis of the hand, hip, and knee. Arthritis Care Res. 2012;64:465-74.

4. Massey T, Derry S, Moore RA, McQuay HJ. Topical NSAIDs for acute pain in adults. Cochrane Database Syst Rev. 2010;CD007402.

5. Moore N, Pollack C, Butkerait P. Adverse drug reactions and drug-drug interactions with over-the-counter NSAIDs. Ther Clin Risk Manag. 2015;11:1061-75.

6. Rannou F, Pelletier J-P, Martel-Pelletier J. Efficacy and safety of topical NSAIDs in the management of osteoarthritis: evidence from real-life setting trials and surveys. Semin Arthritis Rheum. 2016;45:S18-21.

7. Bruyère $\mathrm{O}$, Cooper $\mathrm{C}$, Pelletier J-P, Maheu E, Rannou F, Branco $\mathrm{J}$, et al. A consensus statement on the European Society for Clinical and Economic Aspects of Osteoporosis and Osteoarthritis (ESCEO) algorithm for the management of knee osteoarthritis-from evidence-based medicine to the real-life setting. Semin Arthritis Rheum. 2016;45:S3-11.

8. Pubchem. Loxoprofen [Internet]. [cited 2018 Sep 10]. Available from: https://pubchem.ncbi.nlm.nih.gov/compound/3965.

9. Koo T-S, Kim D-H, Ahn S-H, Kim K-P, Kim I-W, Seo S-Y, et al. Comparison of pharmacokinetics of loxoprofen and its active metabolites after an intravenous, intramuscular, and oral administration of loxoprofen in rats: evidence for extrahepatic metabolism. J Pharm Sci. 2005;94:2187-97.

10. Derry S, Moore RA, Rabbie R. Topical NSAIDs for chronic musculoskeletal pain in adults. Cochrane Database Syst Rev. 2012;CD007400.

11. Sugwara S, Tateishi A, Tanaka M, Kuroki Y, Inoue Y, Iwasaki $\mathrm{Y}$, et al. A dose finding study of a hydrogel patch containing loxoprofen sodium in patients with osteoarthritis of the knee (Late Phase II Clinical Study). J Clin Ther Med. 2006;22:293-310.

12. Sachiko S, Yoshikatsu K, Akio T, Mamoru T, Yukio I, Yoshio I, et al. The effects of LOXONIN PAP $100 \mathrm{mg}$ (hydrogel patch containing loxoprofen sodium) for the treatment of patients with osteoarthritis of the knee-randomized, double-blind clinical study with loxoprofen sodium tablets. J Clin Ther Med. 2006;22:393-409.

13. Tubach F, Ravaud P, Martin-Mola E, Awada H, Bellamy N, Bombardier C, et al. Minimum clinically important improvement and patient acceptable symptom state in pain and function in rheumatoid arthritis, ankylosing spondylitis, chronic back pain, hand osteoarthritis, and hip and knee osteoarthritis: results from a prospective multinational study. Arthritis Care Res. 2012;64:1699-707.

14. Mu R, Bao C, Chen Z, Zheng Y, Wang G, Zhao D, et al. Efficacy and safety of loxoprofen hydrogel patch versus loxoprofen tablet in patients with knee osteoarthritis: a randomized controlled noninferiority trial. Clin Rheumatol. 2016;35:165-73.

15. Schreiber J-U, Mencke T, Pradarutti S, Jeblick S, Fuchs-Buder T. Does preoperatively administered parecoxib prevent succinylcholine-associated myalgia? A randomized, placebo-controlled trial. Eur J Anaesthesiol. 2006;23:332-7.

16. Cohen SP, Verdolin MH, Chang AS, Kurihara C, Morlando $\mathrm{BJ}$, Mao J. The intravenous ketamine test predicts subsequent response to an oral dextromethorphan treatment regimen in fibromyalgia patients. J Pain. 2006;7:391-8. 
17. Gerwin RD. Diagnosing fibromyalgia and myofascial pain syndrome: a guide. J Fam Pract. 2013;62:S19-25.

18. Zhao B, Shi Y, Li Z. Effectiveness and safety of hydrogel patches containing loxoprofen sodium in patients with myalgia: a randomized, controlled, double-blind, double-parallel, multicenter phase 3 trial [abstract no. APLAR-0134]. Int J Rheum Dis. 2013;16(1):1-122.

19. Zhang H, Lin J, Sun T. Double-blind, multicenter trial to evaluate safety and efficacy of hydrogel patch containing loxoprofensodium in treating swelling and pain caused by trauma [abstract no. APLAR-0153]. Int J Rheum Dis. 2013;16(1):1-122.

20. Sugawara S, Kuroki Y, Tanaka M, et al. The effects of Loxonin Pap $100 \mathrm{mg}$ (hydrogel patch containing loxoprofen sodium) for the treatment of myalgia: randomized, double-blind study compared with loxoprofen sodium tablets. J Clin Ther Med. 2006;22:411-26.

21. Sugawara S, Iwasaki Y, Aoki T. The Effects of LOXONIN PAP $100 \mathrm{mg}$ (hydrogel patch containing loxoprofen sodium) for the treatment of swelling or pain following injuries-randomized, double-blind study with loxoprofen sodium tablet. J Clin Ther Med. 2006;22:427-42.

22. Sugawara S, Nobuoka F, Ogawa D, et al. Clinical efficacy of the hydrogel patch containing loxoprofen sodium (LX-A) on osteoarthritis of the knee: a randomized, open label clinical study with ketoprofen patch (phase III therapeutic confirmatory study) [Japanese with English abstract]. J Clin Ther Med. 2007;23(1):55-71.
23. Sugawara S, Nobuoka F, Ogawa D, et al. Clinical efficacy of the hydrogel patch containing loxoprofen sodium (LX-A) on myalgia: a randomized, open label clinical study compared with indometacin patch (phase III therapeutic confirmatory study) [Japanese with English abstract]. J Clin Ther Med. 2007;23(2):127-41.

24. Mizutani H, Miyano F, Uozu A. Drug use result survey of Loxonin Pap (hydrogel patch containing loxoprofen sodium hydrate). J Clin Ther Med. 2010;26:227-40.

25. Mizutani H, Miyano F, Uozu A. Drug use result survey of Loxonin Tape (patch containing loxoprofen sodium hydrate). J Clin Ther Med. 2010;26:727-41.

26. Sawamura R, Sakurai H, Wada N, Nishiya Y, Honda T, Kazui M, et al. Bioactivation of loxoprofen to a pharmacologically active metabolite and its disposition kinetics in human skin. Biopharm Drug Dispos. 2015;36(6):352-63.

27. Sawamura R, Kazui M, Kurihara A, Izumi T. Pharmacokinetics of loxoprofen and its active metabolite after dermal application of loxoprofen gel to rats. Pharm. 2015;70:74-80.

28. Scheiman JM. NSAID-induced gastrointestinal injury: a focused update for clinicians. J Clin Gastroenterol. 2016;50:5-10.

29. Antman EM, Bennett JS, Daugherty A, Furberg C, Roberts H, Taubert KA, et al. Use of nonsteroidal antiinflammatory drugs: an update for clinicians: a scientific statement from the American Heart Association. Circulation. 2007;115:1634-42.

30. Wolfe MM, Lichtenstein DR, Singh G. Gastrointestinal toxicity of nonsteroidal antiinflammatory drugs. N Engl J Med. 1999;340:1888-99.

\section{Affiliations}

\section{Dongbao Zhao ${ }^{1}$ Z Zhiwei Chen ${ }^{2}$ Shaoxian $\mathrm{Hu}^{3}$. Jianhao $\mathrm{Lin}^{4} \cdot$ Zengwu Shao $^{5}$. Guochun Wang ${ }^{6}$. Weiguo Xiao ${ }^{7}$. Yi Zheng ${ }^{8} \cdot$ Zhiyi Zhang $^{9} \cdot$ Yeqing Shi ${ }^{1} \cdot{\text { Zhanguo } \mathrm{Li}^{10}}^{10}$}

1 Department of Rheumatology, Changhai Hospital of Shanghai, Shanghai, China

2 Department of Rheumatology, The First Affiliated Hospital of Soochow University, Suzhou, China

3 Department of Rheumatology, Tongji Hospital, Tongji Medical College, Huazhong University of Science and Technology, Wuhan, China

4 Department of Orthopaedics, Peking University People's Hospital, Beijing, China

5 Department of Orthopaedics, Union Hospital, Tongji Medical College, Huazhong University of Science and Technology, Wuhan, China
6 Department of Rheumatology, China-Japan Friendship Hospital, Beijing, China

7 Department of Rheumatology, The First Hospital of China Medical University, Shenyang, China

8 Department of Rheumatology, Beijing Chao-Yang Hospital, Capital Medical University, Beijing, China

9 Department of Rheumatology, The First Affiliated Hospital of Harbin Medical University, Harbin, China

10 Department of Rheumatology and Immunology, Peking University People's Hospital, No. 11 Xizhimen South Street, Xicheng District, Beijing, People's Republic of China 